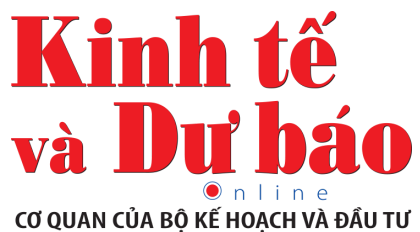

\title{
Năm điều rút ra từ cuốn sách "Trí tuệ nhân tạo: Hướng dẫn về tư duy con người"
}

\author{
$19: 19 \mid 04 / 07 / 2021$
}

Nếu thế giới có thêm những bài báo, những quyển sách như "Artificial Intelligence: A guide for to thinking humans", cuộc trò chuyện về Al sẽ trở nên thu hút và minh bạch.

Trong cuốn sách Artificial Intelligence: A guide for to thinking humans (Tạm dịch: Trí tuệ nhân tạo: Hướng dẫn về tư duy con người), tác giả Melanie Mitchell đã cung cấp một bản đánh giá hiện trạng về các hệ thống trí tuệ nhân tạo (Al) hiện đại trên khắp thế giới, cũng như làm rõ sự khác biệt của những hệ thống này so với cách con người tư duy [1]. Bằng ngôn ngữ đơn giản, gần gũi, Mitchell đã giải thích những thành công và giới hạn của các kĩ thuật Al hàng đầu hiện nay, chẳng hạn như mạng lưới nơ-ron tích chập (convolutional neural networks), mạng nơ-ron hồi quy (regressional neural networks), hay việc học tăng cường sâu (Reinforcement deep-learning)... Ứng dụng của kĩ thuật Al trên có thể tìm thấy trong vô số tiện ích công nghệ chúng ta ngày càng sử dụng trong cuộc sống: phân loại hình ảnh, điều khiển ô tô tự lái, nhận dạng giọng nói, đề xuất tin tức và video, xử lý ngôn ngữ tự nhiên..

Cuốn sách được chia thành các phần tương ứng với các thách thức lớn của Al. Phần I nói về nền tảng của lĩnh vực nghiên cứu Al, phần II nói về thị giác máy tính, phần III bàn về khả năng của $\mathrm{Al}$ trong các trò chơi điện tử, phần IV là về xử lý ngôn ngữ tự nhiên và phần cuối cùng, là về rào cản của ý nghĩa - vấn đề khó khăn nhất đối với Al.

Về tổng thể, cuốn sách có năm điểm thật sự nổi bật. Đầu tiên, hầu hết các hệ thống Al hiện tại đều bắt buộc phải có bộ dữ liệu khổng lồ được "dán nhãn" bởi con người. Từ nhiệm vụ nhận dạng hình ảnh đến xử lý ngôn ngữ tự nhiên, tất cả các hệ thống Al hiện tại đều sử dụng bộ dữ liệu do con người quy định từ định nghĩa dữ liệu, làm sạch và

A PELICAN BOOK

Artificial Intelligence A Guide for Thinking Humans Melanie Mitchell

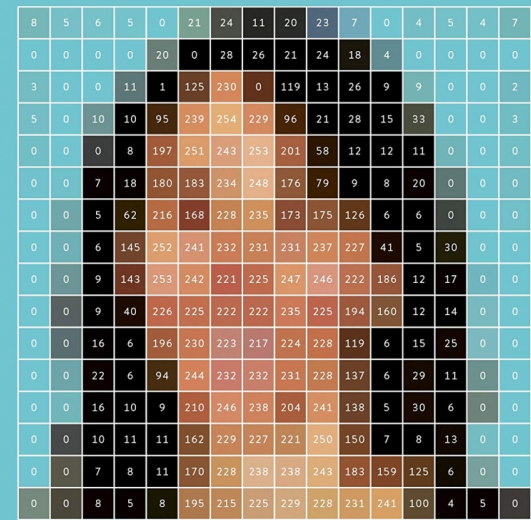

Cuốn sách được chia thành các phần tương ứng với các thách thức lớn của $\mathrm{Al}$ xử lí dữ liệu. Mặc dù bộ dữ liệu chất lượng cao là nền tảng cơ bản của Al và dữ liệu đang được sản xuất và xử lí hàng ngày, nhưng ngành triết lý dữ liệu (philosophy of data) vẫn chưa được định hình cụ thể $[2,3,4]$. Điều này khiến những câu hỏi nền tảng về tính chất bản thể luận của dữ liệu vẫn còn đang bỏ ngỏ.

Ví dụ như, với một vấn đề khoa học bất kì, dù nó được giải quyết bởi con người hay cỗ máy, một nhà nghiên cứu cần phải đặt ra câu hỏi làm thế nào một người hay một cỗ máy có thể xác định dữ liệu của vấn đề, về quy trình vận hành và quản lý dữ liệu của nó... Bên cạnh đó, dữ liệu nên được quy định một cách bản thể học như thế nào trong mối quan hệ với sự kiện, thông tin và kiến thức. Những vấn đề triết học về dữ liệu này vẫn còn hiển hiện và chúng sẽ có ảnh hưởng lớn tới định hướng tư duy của chúng ta về các vấn đề đương đại liên quan tới đạo đức

Dù bề ngoài có cho thấy dấu hiệu của sự thông minh đến mấy, Al cũng không có bất kỳ hiểu biết thực sự nào. Tuy nhiên, giới truyền thông đều đang có xu hướng thổi phồng và đánh giá quá mức năng lực của Al. công nghệ, nhưng dường như đang không có một khuôn khổ thống nhất cho chúng ở thời điểm hiện tại.

Điều này dẫn đến điểm đáng lưu ý thứ hai. Hầu hết trí thông minh trong các hệ thống Al hiện tại có thể được giải thích bằng cách các nhà nghiên cứu $\mathrm{Al}$ điều chỉnh các "siêu tham số" (hyperparameters). "Siêu tham số" là tất cả các khía cạnh yêu cầu sự thiết lập của con người để quá trình học tập có thể bắt đầu: số lần lặp, số lượng tham số, thời điểm khám phá những hướng đi mới, thời điểm nên tiếp tục khai thác các lựa chọn đã được chứng minh là đúng... Đây là công việc khó nhất và cũng là công việc sinh lợi nhất trong lĩnh vực này. Và nếu chúng ta nhìn ở góc 
độ dữ liệu, dường như bất cứ khi nào một hệ thống Al không hoạt động, chúng ta sẽ tìm ra vấn đề ở một điểm nào đó trong cách dữ liệu được xác định hoặc cách dữ liệu được cấu trúc (tham số hóa). Vì vậy, một triết lý thống nhất về dữ liệu có thể giúp làm sáng tỏ các vấn đề liên quan đến siêu tham số.

Thứ ba, tất cả các hệ thống Al đều cho thấy các giới hạn trong tư duy theo những cách không hề giống con người. Nhất là khi đương đầu với các sự kiện hiếm gặp kéo dài, hoặc các bối cảnh không quen thuộc. Một lần nữa, xét từ góc độ dữ liệu, chúng ta đang chờ những đột phá trong kiến giải liên ngành để có hiểu biết đầy đủ về cách con người nhận thức, xử lý dữ liệu và biến chúng thành kiến thức. Vì vậy, thứ suy nghĩ phổ biến, ít nhất là trên các phương tiện truyền thông, rằng việc học của Al hiện tại tương tự như việc học của con người là hoàn toàn sai lệch. Việc báo chí giật gân như vậy cần phải bị chống lại trong truyền thông khoa học và báo chí: cả giới học thuật và truyền thông phải tuân thủ nguyên tắc trí tuệ khiêm tốn, trung thực và cởi mở [5,6].

Thứ tư, đổi mới trong việc phát triển các hệ thống Al này đòi hỏi sự tình cờ may mắn. Nói cách khác, những đổi mới trong cùng một lĩnh vực xa lạ cần phải kết hợp với nhau. Ví dụ, thuật toán ImageNet đã thành công và gây ngạc nhiên cho thế giới Al về tỷ lệ nhận dạng hình ảnh chính xác chính là nhờ người sáng lập, Fei-Fei Li, bằng sự tình cờ, đã vô tình phát hiện ra hai điều. Điều đầu tiên là cơ sở dữ liệu các từ tiếng Anh được tạo ra bởi nhà tâm lý học George Miller, một cơ sở dữ liệu giúp phân loại các danh từ thành các thứ bậc của sự trừu tượng. Điều thứ hai là dịch vụ mới do Amazon cung cấp, Mechanical Turk. Đây là một dịch vụ mà nhân công được thuê để thực hiện các nhiệm vụ tuy dễ dàng đối với con người, nhưng hiện tại lại đang quá khó đối với máy móc: chẳng hạn như gọi tên đồ vật qua hình ảnh. Bên cạnh đó, các ứng dụng Al trong nghệ thuật cũng đặc biệt đáng chú ý [7]. Tương tự như trong thị trường thông thường, lợi thế chiến lược của sự ngẫu nhiên không nên bị đánh giá thấp trong thị trường của những ý tưởng $[8,9]$.

Cuối cùng, như Mitchell (2019) nói một cách ngắn gọn, có một "rào cản về ý nghĩa" trong sự phát triển của Al: dù bề ngoài có cho thấy dấu hiệu của sự thông minh đến mấy, Al cũng không có bất kỳ hiểu biết thực sự nào. Tuy nhiên, giới truyền thông đều đang có xu hướng thổi phồng và đánh giá quá mức năng lực của Al. Ví dụ sinh động nhất là trường hợp của IBM's Watson, khi mà ban đầu, các phương tiện truyền thông chính thống và các kỹ sư đều tuyên bố rằng hệ thống này có thể

Trong việc phát triển các công nghệ mới nhận thức của công chúng không chỉ quan trọng đối với bản thân các công nghệ, mà điều đó còn đóng vai trò then chốt đối với sự tin tưởng vào khoa học.

"đọc" hàng triệu cuốn sách và bài báo. Vậy nhưng, ý nghĩa của từ "đọc" đã được dùng một cách sai lệch. Do đó mà hiện tại, nhiều hợp đồng lớn của IBM's Watson đã bị hủy bỏ. Vậy nên, trong việc phát triển các công nghệ mới, nhận thức của công chúng không chỉ quan trọng đối với bản thân các công nghệ, mà điều đó còn đóng vai trò then chốt đối với sự tin tưởng vào khoa học [10].

Chúng ta cần thêm những quyển sách và các bài báo giống như Artificial Intelligence: $\boldsymbol{A}$ guide for to thinking humans, để cuộc trò chuyện về Al trở nên thu hút và minh bạch hơn. Nói rõ hơn, cộng đồng khoa học cần làm tốt hơn trong ba lĩnh vực: 1) làm sáng tỏ các hệ thống Al mới; 2) tập trung vào các vấn đề kỹ thuật cơ bản về độ tin cậy/độ chính xác, về những thành kiến nội tại, về các rủi ro..., thay vì mối đe dọa của "Al siêu thông minh", 3) thúc đẩy thảo luận về sự cân bằng đạo đức giữa lợi ích và tác hại tiềm ẩn của những hệ thống Al với các bên liên quan.

\section{References}

1. Mitchell, M. (2019). Artificial intelligence: A guide for thinking humans. London: Penguin UK.

2. Napoletani, D., Panza, M., \& Struppa, D. C. (2011). Agnostic science. Towards a philosophy of data analysis. Foundations of Science, 16(1), 1-20.

3. Vuong, Q.-H., et al. (2018). An open database of productivity in Vietnam's social sciences and humanities for public use. Scientific Data, 5(1), 180188.

4. Wickham, H. (2014). Tidy data. Journal of Statistical Software, 59(10), 1-23

5. Vuong, Q. H. (2020). Reform retractions to make them more transparent. Nature, 582(149).

6. Nosek, B. A., \& Errington, T. M. (2020). The best time to argue about what a replication means? Before you do it. Nature, 583(518-520)

7. Ho, M. T. (2021). Công nghệ Al có thể mang lại gì cho nghệ thuật Việt Nam?. Kinh tế và Dự báo. URL: https://kinhtevadubao.vn/cong-nghe-ai-co-the-mang-lai-gi-cho-nghe-thuat-viet-nam-18065.html

8. Napier, N. K., Vuong, Q. H. (2013). Serendipity as a strategic advantage?. In Wilkinson (ed) Strategic Management in the 21st Century (Vol. 1: The Operational Environment), pp. 175-199. Westport, CT: Praeger/ABCClio.

9. Vuong, Q. H., \& Napier, N. K. (2014). Making creativity: the value of multiple filters in the innovation process. International Journal of Transitions and Innovation Systems, 3(4), 294-327.

10. Vuong, Q.-H. (2018). The (ir)rational consideration of the cost of science in transition economies. Nature Human Behaviour, 2(1), 5-5.

Hồ Mạnh Tùng, Viện Triết học, Viện Hàn lâm Khoa học Xã hội Việt Nam

Trần Đức Hưng Long, Đại học Ritsumeikan Asia Pacific University, Beppu, Oita Nhật Bản 
URL: https://kinhtevadubao.vn/nam-dieu-rut-ra-tu-cuon-sach-tri-tue-nhan-tao-huong-dan-ve-tu-duy-con-nguoi-18180.html @ Kinh tế và Dự báo - Bộ Kế hoạch và Đầu tư 